\title{
Comparison of Measurements of Autoantibodies to Glutamic Acid Decarboxylase and Islet Antigen-2 in Whole Blood Eluates from Dried Blood Spots Using the RSR-Enzyme Linked Immunosorbent Assay Kits and In-House Radioimmunoassays
}

\author{
Anders Persson, ${ }^{1}$ Charlotte Becker, ${ }^{2}$ Ida Hansson, ${ }^{3}$ Anita Nilsson, ${ }^{3}$ and Carina Törn ${ }^{1}$ \\ ${ }^{1}$ Unit for Diabetes and Celiac Disease, Department of Clinical Sciences, Lund University, Wallenberg Laboratory, Entrance 46, \\ University Hospital MAS, 20502 Malmö, Sweden \\ ${ }^{2}$ Department of Clinical Chemistry, University Hospital MAS, 20502 Malmö, Sweden \\ ${ }^{3}$ Unit for Diabetes and Celiac Disease, Department of Clinical Sciences, Lund University, Clinical Research Center (CRC), \\ University Hospital MAS, 20502 Malmö, Sweden \\ Correspondence should be addressed to Carina Törn, carina.torn@med.lu.se
}

Received 26 June 2009; Revised 7 September 2009; Accepted 24 March 2010

Academic Editor: Rodica Pop-Busui

Copyright ( $\odot 2010$ Anders Persson et al. This is an open access article distributed under the Creative Commons Attribution License, which permits unrestricted use, distribution, and reproduction in any medium, provided the original work is properly cited.

To evaluate the performance of dried blood spots (DBSs) with subsequent analyses of glutamic acid decarboxylase (GADA) and islet antigen-2 (IA-2A) with the RSR-ELISAs, we selected 80 children newly diagnosed with type 1 diabetes and 120 healthy women. DBSs from patients and controls were used for RSR-ELISAs while patients samples were analysed also with in-house RIAs. The RSR-ELISA-GADA performed well with a specificity of $100 \%$, albeit sensitivity (46\%) was lower compared to in RIA $(56 \% ; P=.008)$. No prozone effect was observed after dilution of discrepant samples. RSR-ELISA-IA-2A achieved specificity of $69 \%$ and sensitivity was lower $(59 \%)$ compared with RIA $(66 \% ; P<.001)$. Negative or low positive patients and control samples in the RSR-ELISA-IA-2A increased after dilution. Eluates from DBS can readily be used to analyse GADA with the RSR-ELISA, even if low levels of autoantibodies were not detected. Some factor could disturb RSR-ELISA-IA-2A analyses.

\section{Introduction}

Sampling of blood as dried blood spots (DBSs) for clinical use is currently used for such diverse diseases as congenital metabolic disorders, diabetes, and HIV infections [1-4]. There are a number of advantages gained when using the DBS-technique with subsequent elution concerning ease of collection, transportation/storage, small blood volumes, and minimal invasiveness compared with serum samples. DBStechnique facilitates sampling procedures since capillary sampling can be used. The capillary blood sampling requires less skill and fewer supplies, it can even be used by diabetes patients at home if they are used to measuring their own blood glucose levels. DBS samples can easily be mailed since there is no risk of leakage. Both transportation and short time storage can be done at room temperature $[5,6]$.
In many countries, all newborn babies are screened for phenylketonuria, galactosemia, congenital hypothyroidism, and other metabolic disorders using dried blood spots, indicating that this technique is suitable for large scale analyses [7-9]. The DBS-samples can be used for both genetic factors (DNA) [10] and proteins (enzymes and antibodies) $[5,11]$.

Five islet autoantibodies are known to characterize type 1 diabetes, namely, islet cell antibodies (ICA), insulin autoantibodies (IAA), glutamic acid decarboxylase antibodies (GADA), islet antigen-2 antibodies (IA-2A), and antibodies against the beta cell specific zink-transporter (ZnT8A) [12]. The first four of these are well characterized and several workshops have been undertaken to standardize the measurement of these autoantibodies in serum [13-15]. Both in-house RIAs and RSR-ELISA kits are well established 
for analyses of GADA as well as for IA-2A. The RSR-ELISA kits show high performance for both GADA and IA-2A in serum samples [15] and $\mathrm{Ca}^{2+}$-treated plasma can also be used [16, 17]. In DASP 2005, both RSR-ELISA-GADA kits and RSR-ELISA-IA-2A kits achieved high sensitivity and specificity [15]. For RSR-ELISA-GADA kits $(n=7)$ sensitivity varied from $84 \%$ to $94 \%$ and specificity from $97 \%$ to $99 \%$ for serum samples. For RSR-IA-2A-ELISA kits, sensitivity ranged from $64 \%$ to $68 \%$ and specificity from $98 \%$ to $100 \%$ for serum samples. In DASP 2005, our in-house RIA-GADA assay gave a sensitivity of $76 \%$ and a specificity of $91 \%$ for serum samples, and our in-house RIA-IA-2A assay gave a sensitivity of $72 \%$ and a specificity of $100 \%$. GADA and IA-2A have been analysed in whole blood eluates with RIA assays with high performance $[18,19]$.

In this study, we wanted to test if GADA and IA-2A can be analysed from whole blood eluates with RSR-ELISAs. If these assays show high performances, the measurements of GADA and IA-2A using ELISA have a potential to be applied in large screening programs for identifying individuals at risk for type 1 diabetes.

\section{Materials and Methods}

2.1. Subjects. Dried blood spots (DBS) were obtained as EDTA-blood spotted onto filter forms (Parajett, Parajett $\mathrm{AB}$, Landskrona, Sweden, with filters from Schleicher and Schuell, Dassel, Germany) and air-dried before transportation to the laboratory. A minimum of $60 \mu \mathrm{L}$ blood was needed to fill the marked circles on the filters.

The study population consisted of a random selection of children with newly diagnosed type 1 diabetes $(n=80$; median age $10 \mathrm{yrs}$; range $2-18 ; \mathrm{M} / \mathrm{F}=1.29$ ) and healthy control women were obtained from five Maternity Clinics in our region as described [20] $(n=120$; median age 32 yrs; range 19-44). The patient's (DBSs) had been stored at room temperature for a median of 56 days (range 8-150) and (DBSs) from controls had been stored for a median of 18 days (range 5-57) before punching with subsequent elution was performed.

All samples were collected under informed consent, as approved by the Ethical Committee at Lund University, Lund.

2.2. Sample Preparation. Discs with a diameter of $6 \mathrm{~mm}$ were punched out with a special punching device (Wallac Delfia dried blood spots puncher prod no. 1296-071, Wallac, Turku, Finland). Four discs were punched out from each specimen into separate wells. A total of $80 \mu \mathrm{L}$ of TBST-buffer ( $150 \mathrm{mmol} / \mathrm{L} \mathrm{NaCl}, 20 \mathrm{mmol} / \mathrm{L}$ Tris, $\mathrm{pH} 7.4$, 0.15\% Tween20, $0.1 \%$ BSA) was added to each well. Samples were left on a plateshaker (Delfia plateshaker 1296-003, Wallac, Turku, Finland) at $500 \mathrm{rpm}$, at $+4^{\circ} \mathrm{C}$ overnight. Next morning, whole blood eluates were spun down $(1 \mathrm{~min}, 1500 \times \mathrm{g}$, Labofuge 400, Heraeus, Langerbold, Germany). Whole blood eluates were pooled into an Eppendorf-micro tube and spun at $10000 \times \mathrm{g}$ for $10 \mathrm{~min}$ to remove cell debris.

\subsection{Assays}

2.3.1. RSR-ELISA Assays. RSR-ELISA kits for GADA (GDE/96) and IA-2A (IAE/96) (RSR Ltd, Pentwyn, Cardiff, UK) were used for analyses of GADA and IA-2A. The assays were performed according to the instructions from the manufacturer, except that whole blood eluates were used in equal amounts as recommended for serum. Optical density was read on an ELISA platereader (E-max, Multical platereader, Molecular Devices Corporation, Menlo Park, CA, USA) at $450 \mathrm{~nm}$, with software Multicalc (Perkin-Elmer, Waltham, MA, USA).

Standards were calibrated against the WHO reference NIBSC (97/550) for the GADA assay [21]. High values (>250 WHO Units/ml) were replaced with 250 for statistical and clinical evaluations. The cut-off level was set to 5 WHO Units/ml for GADA, which is the lowest standard concentration and also the recommended cut-off by the manufacturer for serum samples.

Also for IA-2A, standards were calibrated against the NIBSC (97/550). High values (>400 WHO Units/ml) were replaced with 400 for statistical and clinical evaluations. The cut-off level was set to $15 \mathrm{WHO}$ Units/ml for IA$2 \mathrm{~A}$, which is the lowest standard concentration and also the recommended cut-off by the manufacturer for serum samples. Duplicate sampling including the whole preanalytic procedure was performed in ten subjects for GADA and IA-2A. The coefficient of variation $(\mathrm{CV})$ was calculated as the ratio between standard deviation and mean value for duplicates. The median value of these observations was $6.3 \%$ (range 0.76-13) for GADA in the range of 5.0-46.4 WHO Units/ml. Interassay variation for the same samples in two repetitions was a median $7.7 \%$ (range $1.8 \%-40 \%$ ). The CV for IA-2A was a median $5.0 \%$ (range $0.77-15$ ) in the range 15-25 WHO Units/ml. Interassay variation in two repetitions was a median $46 \%$ (range $25 \%-88 \%$ ).

Samples with a high level of antibodies (GADA or IA$2 \mathrm{~A}$ ) in the in-house RIA, but low in the ELISA were diluted to reveal if this finding could be validated or was due to the prozone effect. The prozone effect is well-known to interfere with titers for ICA $[22,23]$.

2.3.2. In-House RIA for GADA and IA-2A. Aliquots of $30 \mu \mathrm{L}$ of whole blood eluates were obtained using the procedure described in Sample preparation and were added into wells with $30 \mu \mathrm{L}$ of ${ }^{35} \mathrm{~S}$-radiolabelled antigen (GAD65 or IA-2) and incubated overnight on a plateshaker $(500 \mathrm{rpm})$ at $+4^{\circ} \mathrm{C}$. Next morning, plates were spun for $1 \mathrm{~min} 1500 \times \mathrm{g}$. Duplicates of $50 \mu \mathrm{L}$ of the antibody-antigen-complex-solution were added to $50 \mu \mathrm{L}$ of $20 \%$ rProtein A Sepharose Fast Flow (Amersham Biosciences, Uppsala, Sweden) and incubated for 90 minutes at $+4^{\circ} \mathrm{C}$ on a plateshaker $(500 \mathrm{rpm})$. Excess antigen was removed by repeated washing of plates ( 8 times with cold TBST-buffer) using a special washing device (Multiscreen vacuum washer, Millipore, Bedford, MA, USA). Plates were air-dried for $30 \mathrm{~min}$, before addition of $50 \mu \mathrm{L}$ of scintillation liquid (Optiphase Supermix scintillation fluid, PerkinElmer Life Sciences, Boston, MA, USA) to each well. 
The radioactivity was measured in a beta counter (Microbeta counter, PerkinElmer Life Sciences, Boston, MA, USA).

Logaritmic standard curves were used for the GADA assay and IA-2A assay. Our laboratory uses the WHOstandard as local standard. Samples above $50 \mathrm{WHO}$-Units/ml were considered as positive in the GADA assay as were samples above $10 \mathrm{WHO}-$ Units/ml in the IA-2A assay. These cut-off limits were defined using previous results from healthy individuals. A GADA level of $500 \mathrm{WHO}$ Units/ml and an IA-2A level of $250 \mathrm{WHO}$ Units/ml were considered as endpoints for samples analysed with RIA and were not diluted further.

The median CV for duplicates was 3.5\% (range 0-15; $n=$ 29) for GADA and interassay variation was $10.1 \%(n=29)$ for a sample of $35 \mathrm{WHO}$ Units/ml. For another sample of 96 WHO Units/ml, median CV was $3.1 \%$ (range $0-11 ; n=29$ ) and interassay variation was $8.7 \%(n=29)$.

The median CV for duplicates was 3.7\% (range 0-10: $n=$ 30) for IA-2A and interassay variation was $7.8 \%(n=30)$ for a sample of $24 \mathrm{WHO}$ Units/ml. For another sample of 155 WHO Units/ml the median CV was $2.7 \%$ (range $0-11$ : $n=24)$ and interassay variation was $9.7 \%$.

2.3.3. Statistical Analysis. Results are reported as median, interquartile range and minimum and maximum since not normally distributed. If $P$-values were $<.05$ a significant difference was accepted. Wilcoxons signed rank test was used to test for differences in repeated measurements in continuos variables.

McNemars test was used to test for differences in binominal paired observations (positive and negative results).

\section{Results}

3.1. RSR-ELISA-GADA Assay Characteristics for Measurements from Whole Blood Eluates. The threshold of 5.0 WHOUnits/ml corresponded to a specificity of $100 \%$ and a sensitivity of $46 \%(37 / 80$; Table 1$)$. A total of 8 samples showed a concentration of $250 \mathrm{WHO}$ Units/ml or higher, these samples were diluted and showed final concentrations of 222-2460 WHO-Units/ml.

3.2. Comparisons of RSR-ELISA-GADA and an In-House RIA Using Patient's Samples. The RSR-ELISA-GADA achieved lower sensitivity $46 \%$ (37/80) compared with the in-house RIA 56\% (45/80; $P=.008$; Figure 1$)$. All samples $(n=37)$ that were positive in the RSR-ELISA-GADA were also positive in the in-house RIA. The discordant samples were all low level positive samples in the in-house RIA $(n=8$; range 53$125 \mathrm{WHO}$-Units/ml; threshold $50 \mathrm{WHO}-U$ nits/ml). Twelve samples were high when analysed with the in-house RIA (range 198- $\geq 500 \mathrm{WHO}-$ Units/ml) but relatively low in the RSR-ELISA (range 7.3-46 WHO-Units/ml). These samples were checked for prozone effect by dilution but levels were similar after dilution (range $10-52 ; P=.81$ ). There was a correlation in GADA levels in patient's samples found to be positive in both assays $\left(n=37 ; r_{s}=0.82 ; P<.01\right.$; Figure 2$)$. Moreover, GADA levels analysed in double-positive samples
TABle 1: Estimation of positivity for GADA and IA-2A. Number of positive and negative patients ( 80 children newly diagnosed with type 1 diabetes; median age 10; range $2-18$ yrs) and controls (120 healthy women; median 32; range 19-44 yrs) from Sweden.

\begin{tabular}{lcc}
\hline Assay & Positive & Negative \\
\hline RSR-ELISA-GADA & $37(46 \%)$ & $43(54 \%)$ \\
In-house RIA-GADA & $45(56 \%)$ & $35(44 \%)$ \\
\hline \multicolumn{3}{c}{ Controls $(n=120)$} \\
\hline RSR-ELISA-GADA & $0(0 \%)$ & $120(100 \%)$ \\
In-house RIA-GADA & $\mathrm{NA}$ & $\mathrm{NA}$ \\
\hline \multicolumn{3}{c}{ Patients $(n=80)$} \\
\hline RSR-ELISA-IA-2A & $47(59 \%)$ & $33(41 \%)$ \\
In-house RIA-IA-2A & $53(66 \%)$ & $27(34 \%)$ \\
\hline & Controls $(n=120)$ & $83(69 \%)$ \\
\hline RSR-ELISA-IA-2A & $37(31 \%)$ & $\mathrm{NA}$ \\
\hline In-house RIA-IA-2A & $\mathrm{NA}$ &
\end{tabular}

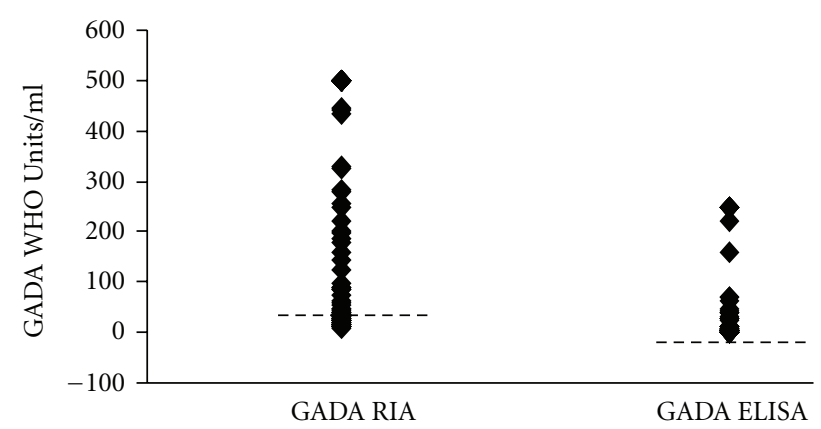

FIGURE 1: GADA levels detected by both ELISA and RIA assays. Cut off levels are indicated by dashed lines.

were higher in the in-house RIA ( $n=37$; median $\geq 500$ WHO Units/ml; interquartile range $235-\geq 500)$ compared to GADA levels analysed with ELISA $(n=37$; median 32 WHO Units/ml; interquartile range $11-189 ; P<.001)$.

3.3. RSR-ELISA-IA-2A Assay Characteristics for Measurements from Whole Blood Eluates. Using the cut-off of 15 WHOUnits/ml the specificity was $69 \%(83 / 120)$ and the sensitivity was $59 \%$ (47/80; Table 1). A total of 16 samples were 400 WHO Units/ml or higher, after dilution the final concentrations of those samples were ranging from 365 to $3430 \mathrm{WHO}-$ Units/ml.

3.4. Comparisons of RSR-ELISA-IA-2A and an In-House RIA Using Patient's Samples. The RSR-ELISA-IA-2A achieved lower sensitivity 59\% (47/80) compared with the in-house RIA 66\% (53/80; $P<.001$; Figure 3). Five low level positive samples in the RSR-ELISA-IA-2A (17-35 WHO Units/ml) were negative in the in-house RIA. Eleven samples were positive only in the in-house RIA (range 17- $\geq 250$ ), six of 


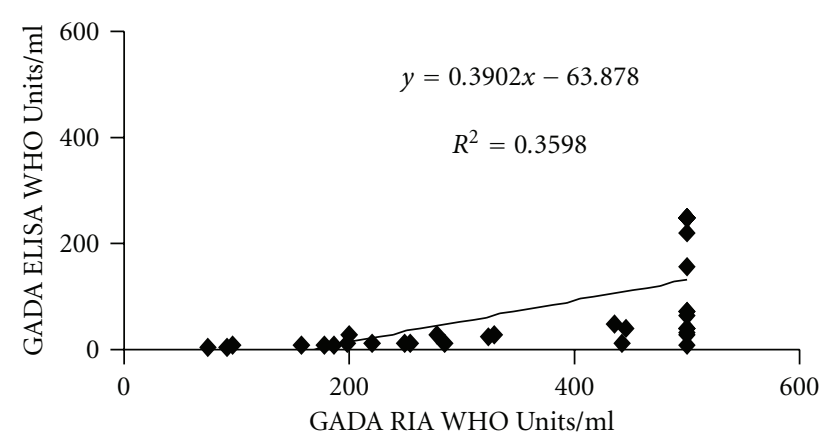

FIGURE 2: GADA levels correlated in patient's samples positive in both the RSR-ELISA and an in-house RIA $\left(n=37 ; r_{s}=0.82\right.$; $P<.01)$.

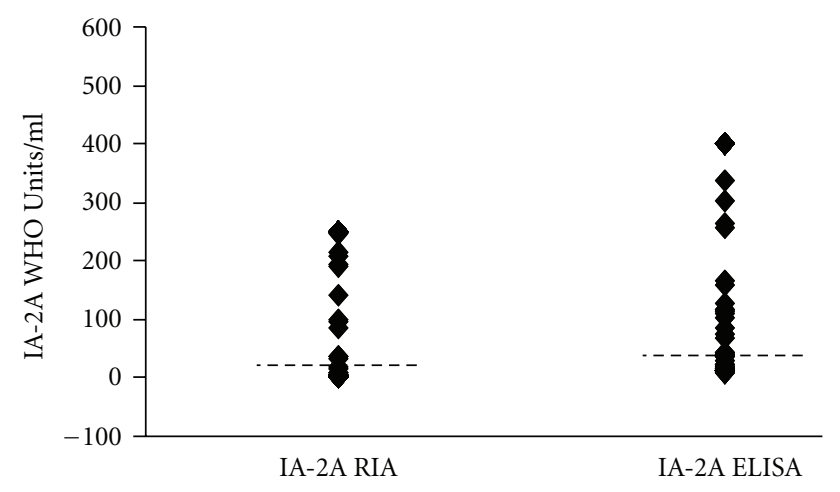

Figure 3: IA-2A levels detected by both ELISA and RIA assays. Cut off levels are indicated by dashed lines.

these samples were 214 WHO Units/ml or higher. We reanalysed two discordant samples that were high level positive in the RIA but negative in the ELISA (with sample material left) and another four samples that were high in the IA-2A RIA ( $n=6$; range 193- $\geq 250$ WHO-Units/ml) but low in the RSR-ELISA ( $n=6$; range 11-36 WHO-Units/ml). After dilution IA-2A levels increased in all samples $(n=6$; range 113-516 WHO-Units/ml; $P=.028$ ) when analysed in the RSR-ELISA-IA-2A. Four patient's samples that were all negative in the in-house RIA, three of whom were low level positive in the RSR-ELISA and one that was negative $(n=4$; range 11-35 WHO-Units $/ \mathrm{ml}$ ) were diluted and reanalysed in the RSR-ELISA and also in this case, levels increased ( $n=$ 4; range 82-888 WHO-Units/ml). Furthermore, 10 control samples found to be clearly negative in the RSR-ELISA $(n=10$; range $8-13 \mathrm{WHO}$-Units $/ \mathrm{ml})$ were also diluted and reanalysed and also in this case levels were higher when diluted (range 29-127 WHO-Units/ml; $P=.005$ ). A total of 42 samples were positive in both assays. There was a weak correlation between IA-2A double positive samples analysed with ELISA and RIA ( $n=42 ; r_{s}=0.52 ; P<.01$; Figure 4$)$. Nevertheless, for IA-2A double positive samples, levels were similar in the in-house RIA $(n=42$; median $\geq 250$ WHO Units/ml; interquartile range $\geq 250-\geq 250$ ) compared with ELISA (median 162 WHO Units/ml; interquartile range 44$\geq 400 ; P=.76)$.

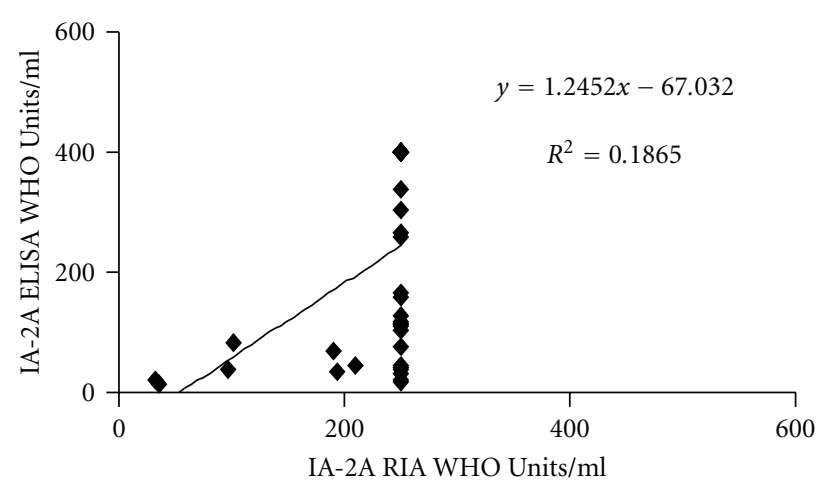

FIGURE 4: A weak correlation was found in IA-2A levels in positive samples both in the RSR-ELISA and an in-house RIA ( $n=42 ; r_{s}=$ $0.52 ; P<.01)$.

3.5. Combined Sensitivity for GADA and IA-2A with In-House RIA and RSR-ELISAAs. Combining the results from GADA and IA-2A measurements with in-house RIAs increased the sensitivity for detecting type 1 diabetes to $79 \%(63 / 80$; $P=$ .0020). Likewise, combining the results from GADA and IA$2 \mathrm{~A}$ measurements with RSR-ELISAs increased the sensitivity to $71 \%(57 / 80 ; P=.0020)$ and expected specificity decreased to $69 \%(83 / 120)$.

\section{Discussion}

In this study, we compared in-house RIAs with commercial ELISAs for analyses of GADA and IA-2A from whole blood eluates both qualitatively and quantitatively. We found that specificity was excellent (100\%) for the RSR-ELISA-GADA, while sensitivity was lower $(46 \%)$ compared with the inhouse RIA (56\%). Discordant samples that were negative in the RSR-ELISA-GADA were low level positive in the in-house RIA. Moreover, GADA levels were lower in the RSR-ELISA in samples found to be positive in both assays. Samples found to have high GADA levels in the in-house RIA, but low in the RSR-ELISA were reanalysed in dilution but levels did not increase. These findings indicate that samples that are positive in the RSR-ELISA-GADA are concordant with measurements in the in-house RIA. However, the RSRELISA failed to detect low level GADA. The lower frequency and lower levels of GADA positive samples can be due to interference of haemoglobin or some other factor in the whole blood. The possibility to measure very high level autoantibodies has little importance in the routine clinical laboratory but can be of interest in intervention studies aimed to decrease levels of autoantibodies [24].

The specificity was very low for RSR-ELISA-IA-2A (69\%) and also the sensitivity was lower for the RSR-ELISA-IA2A (59\%) compared with the in-house RIA (66\%). Among the discordant patient samples, most were high level positive in the in-house RIA. When two of the discordant samples and also four other patient's samples found to be low level positive or negative in the RSR-ELISA-IA-2A were reanalysed in dilution in the RSR-ELISA, levels increased significantly. Also when ten control samples were reanalysed in dilution, 
IA-2A levels increased. We believe that haemoglobin or some other factor in whole blood interfered with the measurement of the antibodies, either via a direct binding to the antigen or antibodies or through a colour shift that affected the optical density, even though we have not fully examined the impact of haemoglobin in this study. It must be borne in mind that these commercial kits are recommended for analyses of autoantibodies in serum. One limitation with our study is that we have not analysed paired serum and DBS samples from patients and controls. However, a similar set of patient serum samples have shown excellent performance for the RSR-ELISA-GADA and IA-2A in our hands [17]. Both our in-house RIAs for GADA and IA-2A achieved high sensitivities $(76 \%$ and $72 \%)$ at excellent specificities in DASP 2005 [15]. RSR-ELISAs for GADA and IA-2A were recently established at the Department of Clinical Chemistry, UMAS, Malmö and have not been subjected to international standardisation. Another possible limitation with our study is that our reference population consisted of only women. Nonetheless, GADA has been shown to be more frequently found in women with type 1 diabetes than in men and also levels are higher in women $[25,26]$. Samples were drawn prior to delivery in these women used as the control population. Pregnancy could decrease immune response, but GADA has been detected at higher frequency among women with gestational diabetes compared with the general population [27]. Therefore, we assume that we have not limited the ability to detect GADA positive subjects in this reference population using the cut-off limits recommended by the manufacturer.

We have not done a specific study on reproducibility over time due to limited amount of specimens. However, GADA and IA-2A autoantibodies are of IgG type [28] as are HIV-antibodies [29]. HIV-antibodies have shown excellent reproducibility for up to six weeks in different storage conditions (room temperature $4^{\circ} \mathrm{C},-20^{\circ} \mathrm{C},-70^{\circ} \mathrm{C}$, and also $37-70^{\circ} \mathrm{C}$ ) [6]. Total $\operatorname{IgE}$ has been also shown to be stable for repeated freeze/thaw cycles [5]. HbAlc, another protein frequently used for analyses with DBStechnique, has shown low (less than 2\%) between-day imprecision for both venous and capillary sampling [1]. Furthermore, our findings indicate that strong haemolysis may interfere with analyses of IA-2A using the RSR-ELISA. It is possible that haemoglobin caused the lower sensitivity for the RSR-ELISA-GADA but specificity was excellent in this case.

In conclusion, the RSR-ELISA can be used for measurement of GADA in whole blood eluates in a reliable manner even if sensitivity is lower compared with an in-house RIA. Some factor could disturb RSR-ELISA-IA-2A analyses.

\section{Acknowledgments}

The Knut and Alice Wallenberg Foundation are acknowledged for a research grant contributing to this study. The authors would like to acknowledge the expert technical assistance from Ulrika Gustavsson and Nils-Göran Persson. Professor Åke Lernmark is thanked for critically reading the manuscript and commenting on it.

\section{References}

[1] J.-O. Jeppsson, P. Jerntorp, L.-O. Almer, R. Persson, G. Ekberg, and G. Sundkvist, "Capillary blood on filter paper for determination of HbA1c by ion exchange chromatography," Diabetes Care, vol. 19, no. 2, pp. 142-145, 1996.

[2] S. Vallian and H. Moeini, "A quantitative bacterial micro-assay for rapid detection of serum phenylalanine in dry blood-spots: application in phenylketonuria screening," Journal of Applied Genetics, vol. 47, no. 1, pp. 79-83, 2006.

[3] A. S. Youngpairoj, A. S. Masciotra, C. Garrido, N. Zahonero, C. de Mendoza, and J. G. Garcia-Lerma, "HIV-1 drug resistance genotyping from dried blood spots stored for 1 year at $4{ }^{\circ} \mathrm{C}$," Journal of Antimicrobial Chemotherapy, vol. 61, no. 6, pp. 1217-1220, 2008.

[4] W. Stevens, G. Sherman, R. Downing, et al., "Role of the laboratory in ensuring global access to ARV treatment for HIVinfected children: consensus statement on the performance of laboratory assays for early infant diagnosis," The Open AIDS Journal, vol. 2, pp. 17-25, 2008.

[5] S. Tanner and T. W. McDade, "Enzyme immunoassay for total immunoglobulin E in dried blood spots," American Journal of Human Biology, vol. 19, no. 3, pp. 440-442, 2007.

[6] A. C. De Castro, L. G. Borges, R. D. S. De Souza, M. Grudzinski, and P. A. D'Azevedo, "Evaluation of the human immunodeficiency virus type 1 and 2 antibodies detection in dried whole blood spots (DBS) samples," Revista do Instituto de Medicina Tropical de Sao Paulo, vol. 50, no. 3, pp. 151-156, 2008.

[7] J. Alm and A. Larsson, "Evaluation of a nation-wide neonatal metabolic screening programme in Sweden 1965-1979," Acta Paediatrica Scandinavica, vol. 70, no. 5, pp. 601-607, 1981.

[8] E. Carreiro-Lewandowski, "Newborn screening: an overview," Clinical Laboratory Science, vol. 15, no. 4, pp. 229-238, 2002.

[9] B. Wilcken and V. Wiley, "Newborn screening," Pathology, vol. 40, no. 2, pp. 104-115, 2008.

[10] W. A. Hagopian, Å. Lernmark, M. J. Rewers, et al., “TEDDYthe environmental determinants of diabetes in the young: an observational clinical trial," Annals of the New York Academy of Sciences, vol. 1079, pp. 320-326, 2006.

[11] Y.-Y. Xu, K. Pettersson, K. Blomberg, I. Hemmila, H. Mikola, and T. Lovgren, "Simultaneous quadruple-label fluorometric immunoassay of thyroid-stimulating hormone, $17 \alpha$ hydroxyprogesterone, immunoreactive trypsin, and creatine kinase MM isoenzyme in dried blood spots," Clinical Chemistry, vol. 38, no. 10, pp. 2038-2043, 1992.

[12] J. M. Wenzlau, K. Juhl, L. Yu, et al., "The cation efflux transporter ZnT8 (Slc30A8) is a major autoantigen in human type 1 diabetes," Proceedings of the National Academy of Sciences of the United States of America, vol. 104, no. 43, pp. 17040-17045, 2007.

[13] C. F. Verge, D. Stenger, E. Bonifacio, et al., "Combined use of autoantibodies (IA-2 autoantibody, GAD autoantibody, insulin autoantibody, cytoplasmic islet cell antibodies) in type 1 diabetes. Combinatorial islet autoantibody workshop," Diabetes, vol. 47, no. 12, pp. 1857-1866, 1998.

[14] P. J. Bingley, E. Bonifacio, and P. W. Mueller, "Diabetes antibody standardization program: first assay proficiency evaluation," Diabetes, vol. 52, no. 5, pp. 1128-1136, 2003.

[15] C. Törn, P. W. Mueller, M. Schlosser, E. Bonifacio, and P. J. Bingley, "Diabetes antibody standardization program: evaluation of assays for autoantibodies to glutamic acid decarboxylase and islet antigen-2," Diabetologia, vol. 51, no. 5, pp. 846-852, 2008. 
[16] E. Nilson, B. Ekholm, B. Rees Smith, C. Törn, and M. Hillman, "Calcium addition to EDTA plasma eliminates falsely positive results in the RSR GADAb ELISA," Clinica Chimica Acta, vol. 388, no. 1-2, pp. 130-134, 2008.

[17] K. Rahmati, Å. Lernmark, C. Becker, et al., "A comparison of serum and EDTA plasma in the measurement of glutamic acid decarboxylase autoantibodies (GADA) and autoantibodies to islet antigen-2 (IA-2A) using the RSR radioimmunoassay (RIA) and enzyme linked immunosorbent assay (ELISA) kits," Clinical Laboratory, vol. 54, no. 7-8, pp. 227-235, 2008.

[18] E. Bazzigaluppi, R. Bonfanti, P. J. Bingley, E. Bosi, and E. Bonifacio, "Capillary whole blood measurement of islet autoantibodies," Diabetes Care, vol. 22, no. 2, pp. 275-279, 1999.

[19] C. Wasserfall, M. Atkinson, E. Jodoin, et al., "Glutamic acid decarboxylase and IA-2 autoantibodies in type 1 diabetes: comparing sample substrates for autoantibody determinations," Pediatric Diabetes, vol. 1, no. 1, pp. 10-16, 2000.

[20] K. F. Lynch, B. Lernmark, J. Merlo, C. M. Cilio, S.-A. Ivarsson, and A. Lernmark, "Cord blood islet autoantibodies and seasonal association with the type 1 diabetes high-risk genotype," Journal of Perinatology, vol. 28, no. 3, pp. 211-217, 2008.

[21] A. R. Mire-Sluis, R. Gaines Das, and A. Lernmark, “The World Health Organization international collaborative study for islet cell antibodies," Diabetologia, vol. 43, no. 10, pp. 1282-1292, 2000.

[22] M. Landin Olsson, G. Sundkvist, and A. Lernmark, "Prolonged incubation in the two-colour immunofluorescence test increases the prevalence and titres of islet cell antibodies in type 1 (insulin-dependent) diabetes mellitus," Diabetologia, vol. 30, no. 5, pp. 327-332, 1987.

[23] P. G. Colman, U. Di Mario, A. Rabizadeh, F. Dotta, E. Anastasi, and G. S. Eisenbarth, "A prozone phenomenon interferes in islet cell antibody detection: direct comparison of two methods in subjects at risk of diabetes and in insulin dependent diabetics at onset," Journal of Autoimmunity, vol. 1, no. 2, pp. 109-117, 1988.

[24] G. Sundkvist, W. A. Hagopian, M. Landin-Olsson, et al., "Islet cell antibodies, but not glutamic acid decarboxylase antibodies, are decreased by plasmapheresis in patients with newly diagnosed insulin-dependent diabetes mellitus," Journal of Clinical Endocrinology and Metabolism, vol. 78, no. 5, pp. 1159-1165, 1994.

[25] R. S. Schmidli, H. J. DeAizpurua, L. C. Harrison, and P. G. Colman, "Antibodies to glutamic acid decarboxylase in at-risk and clinical insulin-dependent diabetic subjects: relationship to age, sex and islet cell antibody status, and temporal profile," Journal of Autoimmunity, vol. 7, no. 1, pp. 55-66, 1994.

[26] P. Z. Zimmet, R. B. Elliot, I. R. Mackay, et al., "Autoantibodies to glutamic acid decarboxylase and insulin in islet cell antibody positive presymptomatic type 1 diabetes mellitus: frequency and segregation by age and gender," Diabetic Medicine, vol. 11, no. 9, pp. 866-871, 1994.

[27] C. Nilsson, D. Ursing, C. Törn, A. Åberg, and M. LandinOlsson, "Presence of GAD antibodies during gestational diabetes mellitus predicts type 1 diabetes," Diabetes Care, vol. 30, no. 8, pp. 1968-1971, 2007.

[28] M. I. Hawa, D. Fava, F. Medici, et al., "Antibodies to IA-2 and GAD65 in type 1 and type 2 diabetes: isotype restriction and polyclonality," Diabetes Care, vol. 23, no. 2, pp. 228-233, 2000.
[29] M. Raux, L. Finkielsztejn, D. Salmon-Ceron, et al., "Development and standardization of methods to evaluate the antibody response to an HIV-1 candidate vaccine in secretions and sera of seronegative vaccine recipients," Journal of Immunological Methods, vol. 222, no. 1-2, pp. 111-124, 1999. 


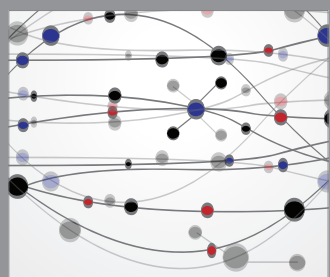

The Scientific World Journal
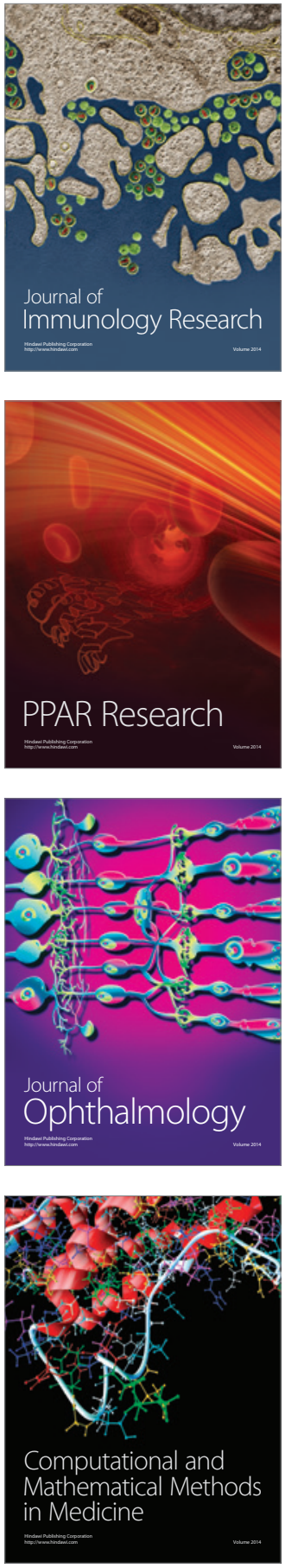

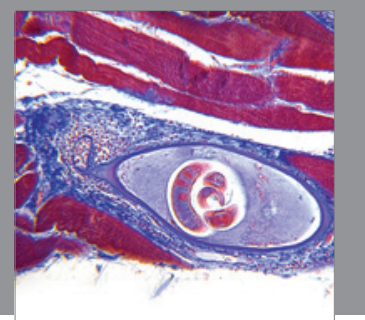

Gastroenterology

Research and Practice
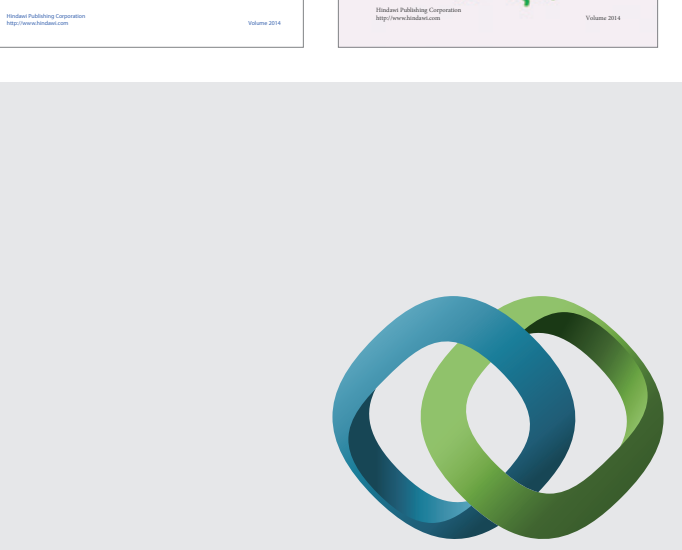

\section{Hindawi}

Submit your manuscripts at

http://www.hindawi.com
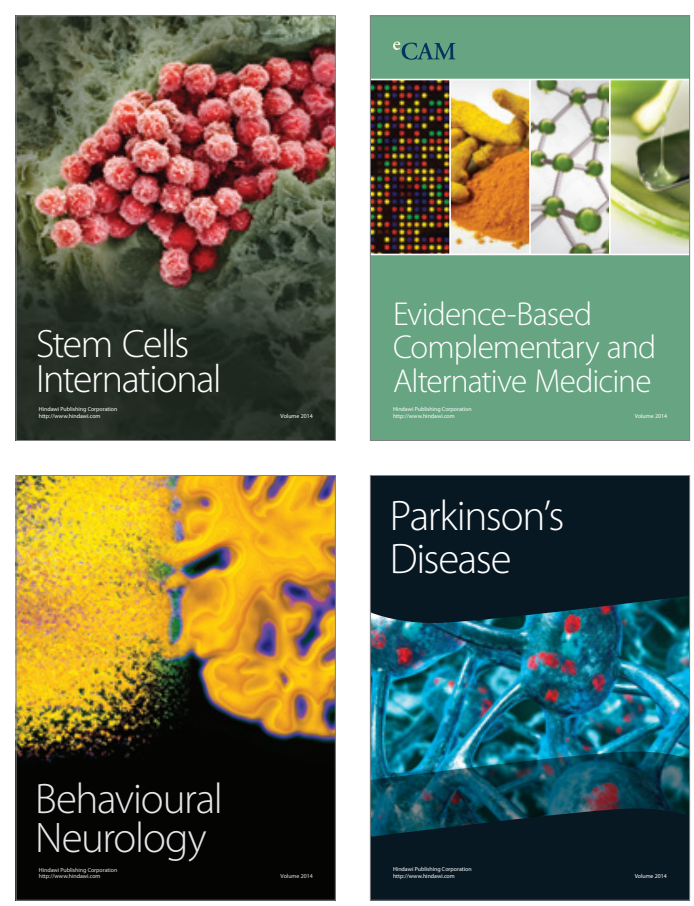

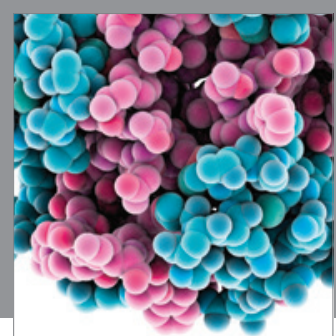

Journal of
Diabetes Research

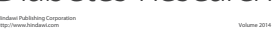

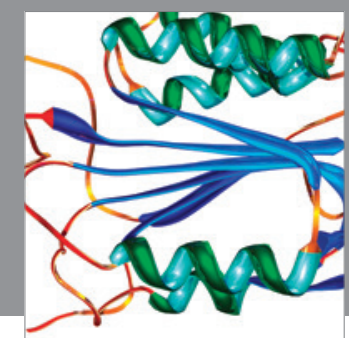

Disease Markers
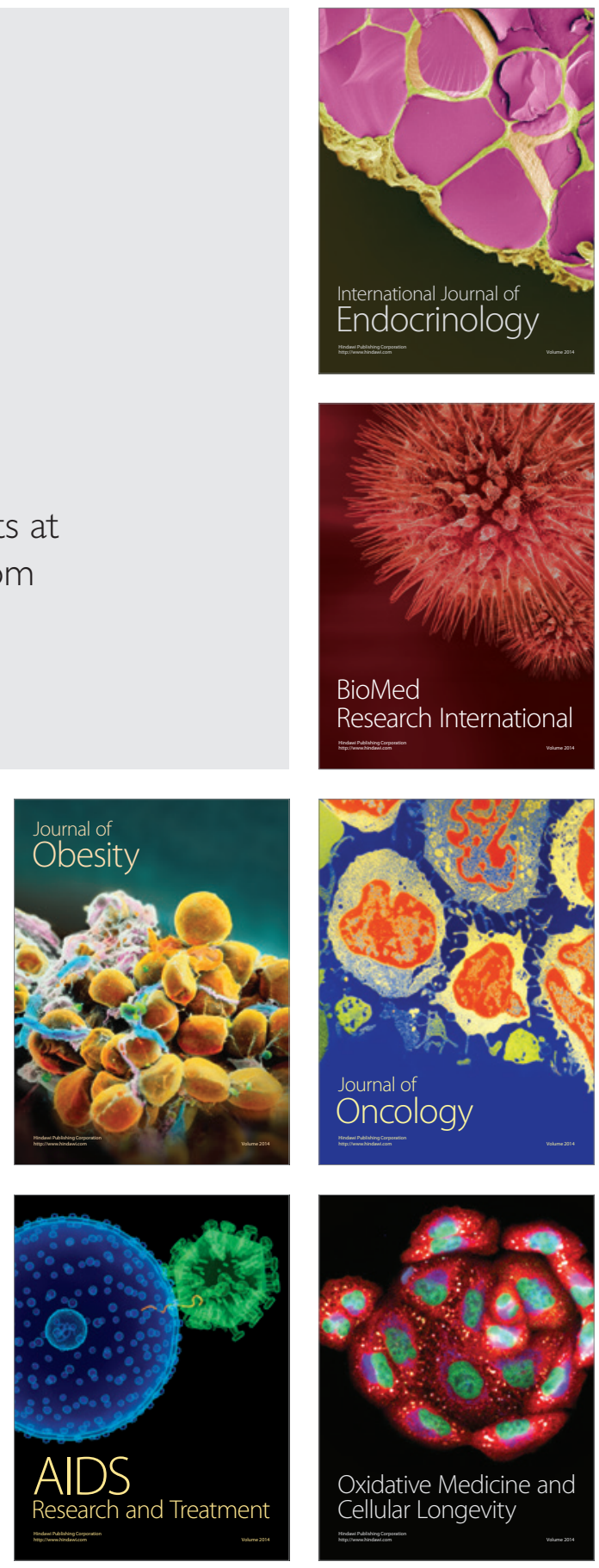